\title{
Structural Failure Analysis and Numerical Simulation of Micro- accelerometers under Impulsive Loading
}

\author{
Yu-Qun Hu ${ }^{1,2}$, Li-Sen Wang ${ }^{1}$, Zhao-Jun Zeng ${ }^{3}$, Yi-Long Hao ${ }^{3}$, Ya-Pu Zhao ${ }^{1}$
}

1. State Key Laboratory of Nonlinear Mechanics, Institute of Mechanics, Chinese Academy of Sciences, Beijing 100080, China,Email: yzhao@lnm.imech.ac.cn

2. College of Aerospace Engineering, Nanjing University of Aeronautics and Astronautics, Nanjing 210016, China

3. Institute of Microelectronics, Peking University, Beijing 100871, China

\begin{abstract}
Micromachined accelerometer is a kind of inertial MEMS devices, which usually operate under intensive impact loading. The reliability of micromachined accelerometers is one of the most important performance indices for their design, manufacture and commercial application. Therefore, great concern has been aroused for the overload reliability of such devices. In the present paper, drop tests and some structural failure analyses have been implemented for a kind of newly designed microaccelerometer fabricated by the standard bulk-machining process of single crystal silicon, and numerical simulation of structural failure behaviour has been carried out for the new device subjected to impulsive loading.
\end{abstract}

Keywords: Micromachined accelerometer, drop test, failure analysis, numerical simulation

\section{Introduction}

During the past decades, there have been many progresses in the design and fabrication of micro-electro-mechanical systems (MEMS). At present, among the numerous applications involving MEMS technology, micromachined inertial sensor devices have been playing an important role. Micromachined accelerometer is one kind of micromachined inertial sensors and it alone has the second largest sales volume after pressure sensors[1]. This is due to their low-cost, small size, light-weight, and the large demand for civilian and military applications.

Although MEMS technology has achieved tremendous progress in the past years, from a historical perspective, MEMS technology is relatively new and is in many ways not mature[2]. The promising applications of MEMS devices are great, but the utilization of them, especially in the applications for military and aerospace systems, have been limited so far due to concerns of reliability and qualifiability of MEMS devices. The greatest challenge for the successful commercialization of MEMS devices is in providing their reliability at various loading environments (including vibration and shock)[3]. Until now, most of the researches on MEMS devices have focused on developing advanced fabrication techniques and improving their functional performance. It is only in the past few years when MEMS technologies and device performance have advanced sufficiently and the applications have become so critical that researchers have paid more attention to the issues of reliability and long-term survivability [4]. In order to ensure the safe applications of those MEMS devices under the extreme cases of loading which they maybe experience, the structural failure analysis and dynamic drop test would be expected.

In this paper, drop tests and some structural 
failure analyses will be implemented for a kind of newly designed microaccelerometer structure fabricated by the standard bulk-machining process of single crystal silicon. Numerical simulation of failure behaviour will be carried out for the new microstructure subjected to impulsive loading.

\section{Design and Fabrication}

Usually, there are two prevailing methods for the fabrication of micromachined inertial accelerometers. One is the surface-machining process and the other is the bulk-machining process. The former is an additive process in which thin films of typically poly-silicon and silicon-oxide are grown on a wafer, and while the latter is a subtractive process in which silicon is removed by wet or dry-etching techniques and forms a proof mass and a suspension structure[5]. Surface-machining process is compatible with a standard CMOS process and can make the microstructures integrated with the circuitry interface on a single chip, but the structure has been limited in size of proof mass, which would restrict the sensitivity of accelerometers[6]. In addition, the sense capacitance of the microaccelerometers has also been limited with the surface machining process [7]. Therefore, our newly designed microaccelerometer has been fabricated by bulk-machining process.

The microaccelerometer is shown as Figure 1 and consists of large proof mass, folded flexible beam, comb finger capacitors and metal electrodes. The proof mass is suspended by two folded flexure silicon beams that act as spring components. There are two groups of combs constructed respectively on the both sides of the proof mass, and with the combs opposite to them, these two groups of combs could form two differential sense capacitors. The proof mass is the middle electrode while the two rows of combs, which have been fixed on top and bottom glass substrate, act as the fixed electrodes. The sense direction is in the proof mass plane. The comb structures can provide enough capacitance by adjusting the number of the comb fingers. In the type shown in Figure 1, the number of comb fingers is taken to be 105 for the proof mass, and 106 for the comb fingers fixed on the base respectively. The calculated initial capacitance of the microaccelerometer is about 1.13 pf [6].

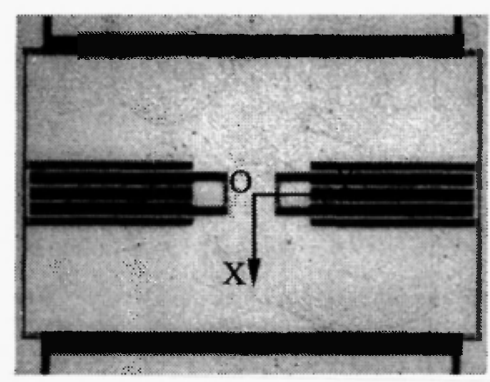

Figure 1. Plan form of Microaccelerometer

\section{Drop Tests of Microaccelerometer}

Usually, microaccelerometers often operate in dynamic environment. For proving the reliability of microaccelerometer structure, some drop tests have been completed. In the following, two kinds of microaccelerometers with the beams of different width have been tested in the experiments. Figure 1 has shown the coordinate system during the drop testing. The sense direction is along $\mathrm{X}$-axis.

Table 1. Experimental results for thin beam microaccelerometers from $1.5 \mathrm{~m}$

\begin{tabular}{ccccc}
\hline $\begin{array}{c}\text { Output } \\
\text { (mV) }\end{array}$ & $\begin{array}{c}\text { Acceleration } \\
\text { G }\end{array}$ & X-axis & Y-axis & Z-axis \\
\hline 512 & 5120 & + & + & - \\
524 & 5240 & + & + & - \\
556 & 5560 & - & - & - \\
576 & 5760 & - & + & - \\
\hline
\end{tabular}

\subsection{Drop test of microaccelerometer with the beams of $6 \mu \mathrm{m}$ width}

In this case, the test specimens dropped to a target of cast iron from height of $1.5 \mathrm{~m}$ with the impact direction of $\mathrm{X}$-axis, $\mathrm{Y}$-axis, and $\mathrm{Z}$-axis respectively, and the experimental results have been listed in Table 1. The output signal can be acquired by a standard accelerometer which has been installed together with the specimens and 
has a resolution of $0.1 \mathrm{mV} / \mathrm{g}$. In Table 1, “_" denotes a structural failure for the tested specimen, and " + " denotes no destruction on them.

\subsection{Drop test of microaccelerometer with the beams of $12 \mu \mathrm{m}$ width}

The test specimens with beams of $12 \mu \mathrm{m}$ dropped to the target from heights of $1.5 \mathrm{~m}$ and $3 \mathrm{~m}$, according to the impact direction of $\mathrm{X}$-axis, $\mathrm{Y}$-axis, and $\mathrm{Z}$-axis respectively. The experimental results have been shown in Tables 2 and 3.

Table 2. Experimental results for thick beam microaccelerometers from $1.5 \mathrm{~m}$

\begin{tabular}{ccccc}
\hline $\begin{array}{c}\text { Output } \\
(\mathrm{mV})\end{array}$ & $\begin{array}{c}\text { Acceleration } \\
\mathbf{G}\end{array}$ & $\mathrm{X}$-axis & Y-axis & Z-axis \\
\hline 464 & 4640 & + & + & + \\
546 & 5460 & + & + & - \\
573 & 5730 & + & + & + \\
\hline
\end{tabular}

Table 3. Experimental results for thick beam microaccelerometers from $3 \mathrm{~m}$

\begin{tabular}{ccccc}
\hline $\begin{array}{c}\text { Output } \\
(\mathrm{mV})\end{array}$ & $\begin{array}{c}\text { Acceleration } \\
\mathbf{U}\end{array}$ & $\mathrm{X}$-axis & Y-axis & Z-axis \\
\hline 2320 & 23200 & + & + & - \\
2600 & 26000 & + & + & + \\
3400 & 34000 & + & + & - \\
\hline
\end{tabular}

From the result data, it can be found that the destruction would occur for the specimens with beams of $6 \mu \mathrm{m}$ width if in the sense direction, i.e. the direction of $\mathrm{X}$-axis, the impact intensities exceed the limit about 5,000g, and for those with beams of $12 \mu \mathrm{m}$ width, the destruction would appear at a limit beyond the value above. The more details on these drop tests are referred in the Ref. [7].

\section{Failure Analyses and Simulation}

The results of drop tests indicate that these MEMS devices much more incline to structural failures when impact loading occurs in positive $\mathrm{Z}$-axis direction than in other directions. The most of structural damages have occurred near the fixed end of the cantilever beams and one of these typical examples have been shown in Figure 2(a). Nevertheless, not a little of ruptures have appeared somewhere along the spans of those beams.

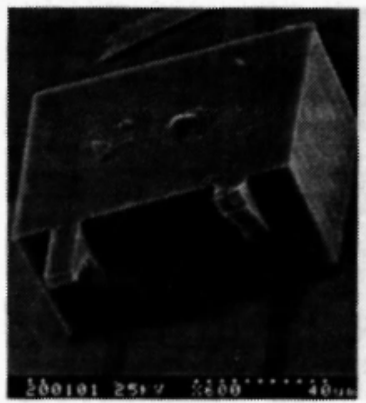

(a)

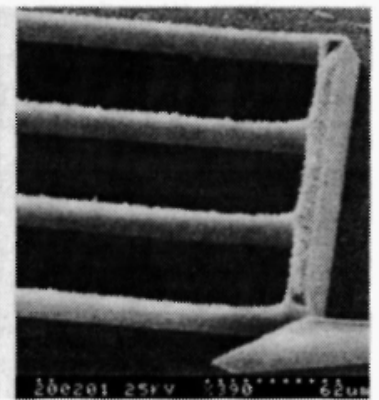

(b)
Figure 2. SEM after drop testing

Wang et al. have presented some preliminary quasi-static theoretical failure analyses for microaccelerometers subjected to impact loading [7]. Here some numerical analyses and simulations have been put forward for the above micro devices under the impact environment. Considering the symmetry of the microaccelerometers, the numerical model has been constructed as shown in Figure 3. Furthermore, the proof mass is simplified as a mass block installed on the free end of the folded beams.

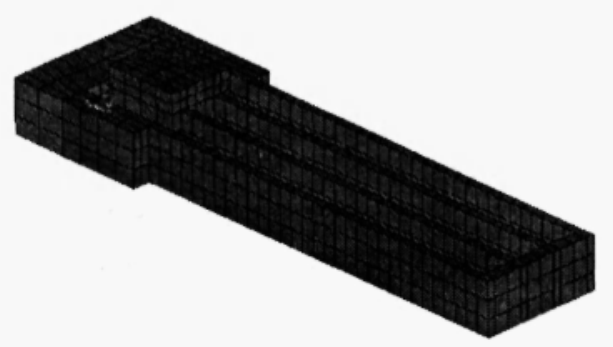

Figure 3. Numerical analysis model

The numerical simulations demonstrate that, in every instant, with the impact loading from Zaxis direction, the maximum Von Mises stress always occurs at the top and bottom sides of the micro beams. With shock time lasting and the propagation of the stress waves within the beams, the locations of maximum Von Mises equivalent stress are traveling in the spans of the beams along their both sides, which has been 
shown in Figure 4. When there exist some bad quality surfaces on the micro beams, e.g. some initial faults or bigger roughness, which often come from the fabrication processes, such as shown in Figure 2(b), fractures would occur more easily in the areas of stress concentration due to maximum stress wave front, and these cases result in the structural failures ultimately.

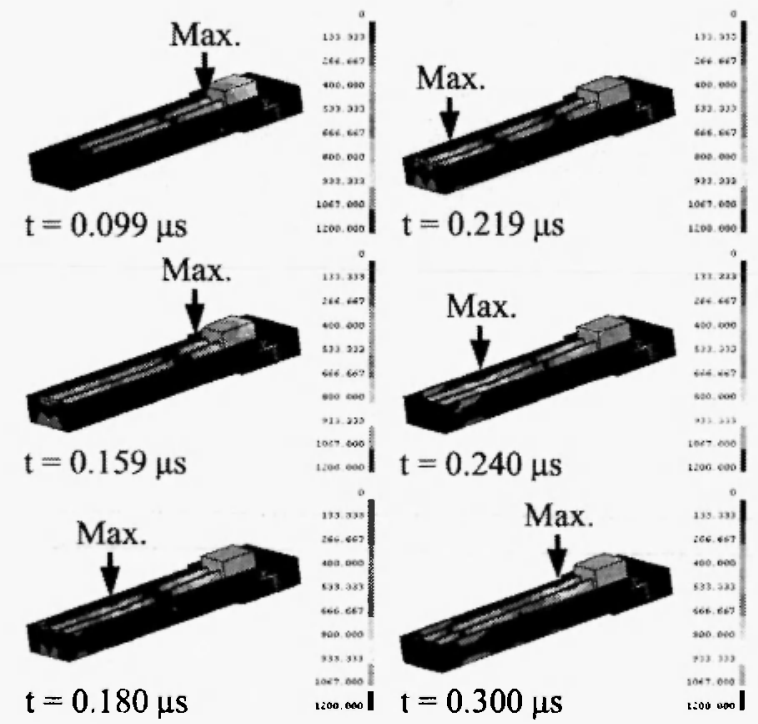

Figure 4. Propagation of Max. Von Mises stress

\section{Summary}

Microaccelerometers have been constructed by silicon-based materials. From the SEM pictures of tested devices, material of this kind demonstrated the fracture with characteristic of brittleness when they are under intensive impact loading. The strength of practical microaccelerometer structures are limited by many unpredictable factors that come from the fabrication processes. These disadvantage factors frequently result in the larger deviations between the ideal designs and the practical devices. Nevertheless, numerical simulation can provide some helpful clues for structural failure analysis of microaccelerometers.

To have a better understanding of the failure mechanisms of microaccelerometers under impact loading, the dynamic failure process is to be studied. It is found that microstructure has a major influence on wave propagation[8]. To predict the dynamic behavior of MEMS, a higher-order dynamic theory is to be developed [9].

\section{Acknowledgments}

This work was supported by the National Natural Science Foundation of China, China National "973" Project, and Key Project of Chinese Academy of Sciences (Grant No. KJCX2-SW-L2).

\section{References}

[1] Yazdi, N., Ayazi, F., Najafi, K.. Micromachined inertial sensors. Proceeding of the IEEE, 86(8) 1640-1659(1998).

[2] Li, G.X., Shemansky, F.A. Drop test and analysis on micro-machined structures, Sensors and Actuators, 85: 280-286 (2000).

[3] Zhao, Y.P., Yu, T.X. Failure modes of MEMS and microscale adhesive contact theory, Int. J. Nonl. Sci. Num. Simul., 1(1) 361-372(2000).

[4] Man, K.F. MEMS reliability for space applications by elimination of potential failure modes through testing and analysis, 1999 SPIE Symp. on Micromachining and Microfabrication, Santa Clara, California, Sept. 21-23, 1999.

[5] Kraft, M. Micromachined inertial sensors state of the art and a look into the future, IMC Measurement and Control, 33(6) 164-168(2000).

[6] Wang, L.S., Zhao, Y.P., Li, Z.H., et al. A bulk micro-machined accelerometer with comb fingers sense capacitors, Int. J. Nonl. Sci. Num. Simul., 1(1) 431-436(2000).

[7] Wang, L.S., Hu, Y.Q., Li, Z.H., Yu, T.X., Zhao, Y.P. Failure analysis of microaccelerometer under impact loading, J. Mechanical Strength, 23(4) 516-522(2001). (In Chinese)

[8] Zhao, Y.P., Zhao, H., Hu, Y.Q. Stress wave propagation in a gradient elastic medium, Chin. Phys. Lett., 19(7) 950-952(2002).

[9] Zhao, Y.P. Some basic problems of microdynamics of solids. In: Engineering Plasticity and Impact Dynamics (L. Zhang Ed.), World Scientific, 261-278 (2001). 